\title{
COMPLETE ALPHA-TENSOR FOR SOLAR DYNAMO
}

\author{
V.N. KRIVODUBSKIJ \\ Astronomical Observatory of Kiev University, Kiev 254053, Ukraine \\ and
}

M. SCHULTZ

Astrophysikalisches Institut Potsdam, An der Sternwarte 16, Potsdam D-O-1591, Germany

\begin{abstract}
We applied the results of recent derivations of the $\alpha$-effect to the solar convection zone model by Stix (1989) to find the depth and latitude dependences of the $\alpha$-tensor. Pronounced inhomogeneity and anisotropy are revealed: the $\alpha$-coefficients for different directions are quite different and strongly latitude-dependent.
\end{abstract}

Knowledge of the complete tensoral representation of the $\alpha$-effect is important to develope stellar dynamos. The expressions for the coefficients $\alpha_{n}$ of the $\alpha$-tensor,

$$
\alpha_{i j}=\alpha_{1}(\mathrm{~g} \cdot \Omega) \Omega^{-1} \delta_{i j}+\alpha_{2}\left(g_{i} \Omega_{j}+g_{j} \Omega_{i}\right) / \Omega+\alpha_{4}(\mathrm{~g} \cdot \Omega) \Omega_{i} \Omega_{j} / \Omega^{3},
$$

have recently become available as fully-nonlinear functions of the angular velocity (Rüdiger and Kichatinov, 1993). In the Eq. (1), $\mathrm{g}$ is the radial unit vector and $\boldsymbol{\Omega}$ is the angular velocity. The tensor (1) has five non-zero components in the spherical coordinates system, $r, \theta, \phi$ :

$$
\begin{gathered}
\alpha_{\phi \phi}=\alpha_{1} \cos \theta, \alpha_{r r}=\left(\alpha_{1}+2 \alpha_{2}\right) \cos \theta+\alpha_{4} \cos ^{3} \theta \\
\alpha_{\theta \theta}=\alpha_{1} \cos \theta+\alpha_{4} \sin ^{2} \theta \cos \theta, \alpha_{r \theta}=\alpha_{\theta r}=-\alpha_{2} \sin \theta-\alpha_{4} \cos ^{2} \theta \sin \theta .
\end{gathered}
$$

All the coefficients $\alpha_{n}$ are superpositions of the contributions made by the two basic inhomogeneities of density and the turbulence intensity: $\alpha_{n}=\alpha^{\text {dens }}+\alpha^{\text {int }}$. We used the solar convection zone model by Stix (1989) to evaluate the $\alpha$-tensor components. The results are shown in the figures 1 to 3 .

Only $\alpha_{\phi \phi}$-component is important for the $\alpha \Omega$-dynamos. Fig.1 shows that the inhomogeneities of density and the turbulence intensity contribute this component with opposite signs. The $\alpha_{\phi \phi}$ varies rather slowly with depth except for the nearbottom layer where strong intensity gradient makes the $\alpha_{\phi \phi}$ to change its sign.

The often used isotropic representation, $\alpha_{i j}=\alpha_{0} \cos \theta \delta_{i j}$, implies the same depth-dependences for all components of the $\alpha$-tensor and the independence of $\alpha / \cos \theta$ of latitude. Our findings are in drastic contrast to the isotropic picture. The depth-dependences shown in the Figures 1 and 2 are quite different one from another and display pronounced variations from pole to equator. Next, Fig. 3 shows that the $\alpha$-tensor possesses the off-diagonal component which is also a manifestation of anisotropy.

It is very tempting to look at the consequences of the strongly anysotropic $\alpha$ effect for solar dynamo. Some preliminary results are presented by Elstner and Rüdiger (1993). 


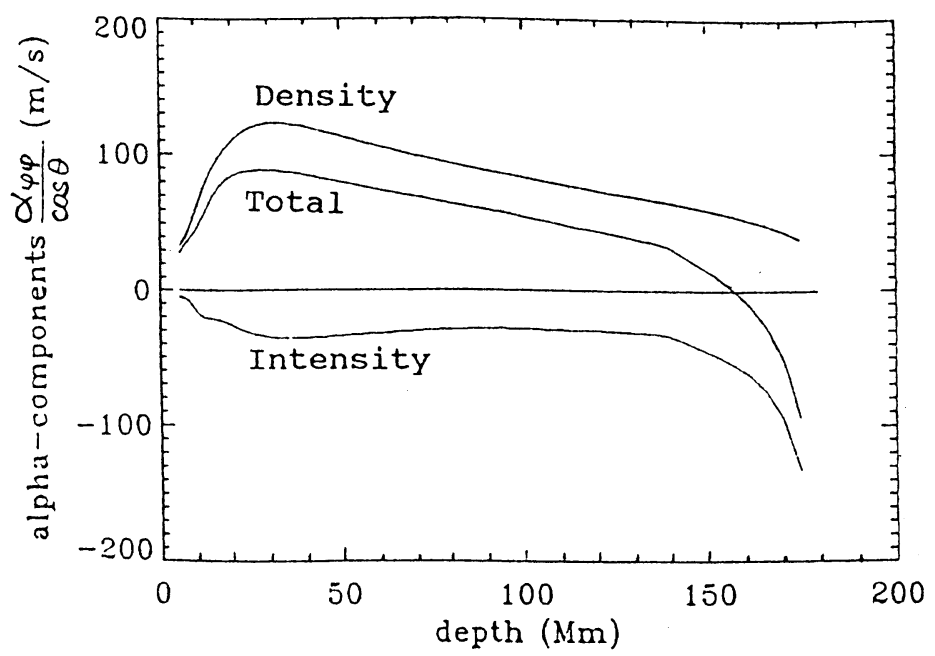

Fig. 1. The total value of $\alpha_{\phi \phi} / \cos \theta$ and its constituents due to inhomogeneities of density and the turbulence intensity as functions of depth in the SCZ
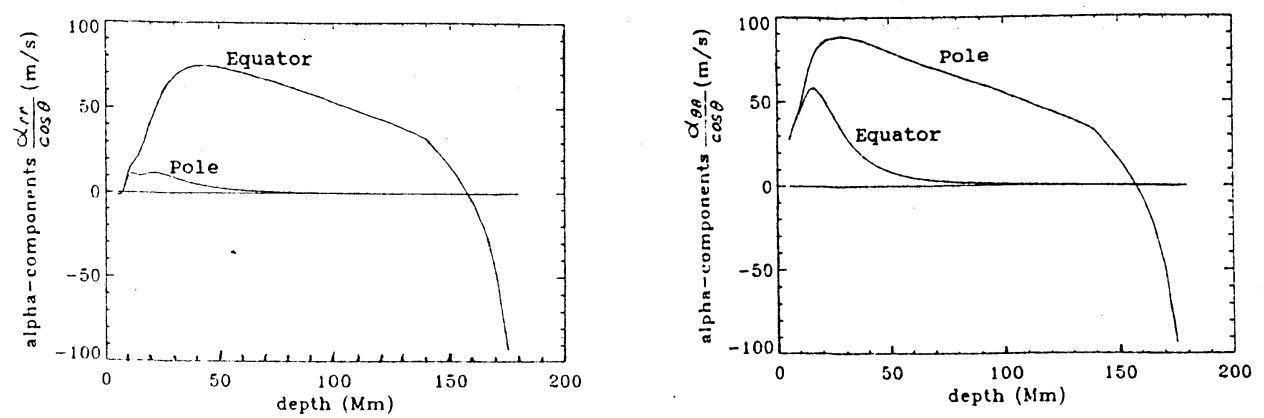

Fig. 2. The total values of $\alpha_{r r} / \cos \theta$ (left) and $\alpha_{\theta \theta} / \cos \theta$ (right) as functions of depth in the SCZ at the pole and the equator

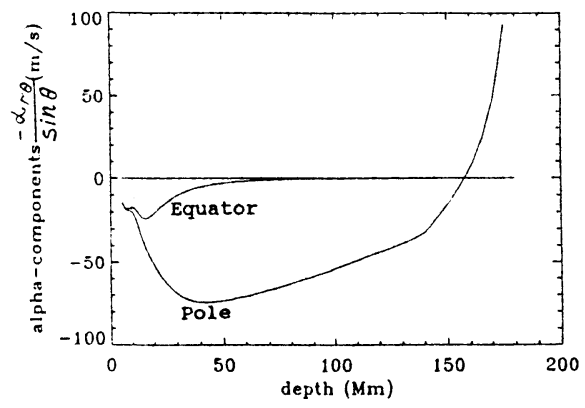

Fig. 3. The same as in Fig.2 but for the off-diagonal component, $-\alpha_{r \theta} / \sin \theta$

\section{References}

Elstner, D. and Rüdiger, G.: 1993, This volume

Rüdiger, G. and Kichatinov, L.L.: 1993 Astron. Astrophys. (in press)

Stix, M.: 1989, The Sun: An Introduction, Springer, Berlin Heidelberg 